\title{
Somatic mutations in genes associated with mismatch repair predict survival in patients with metastatic cancer receiving immune checkpoint inhibitors
}

\author{
YONGPING LIU ${ }^{1-3}$, LIN CHEN $^{3,4}$, SHENLI ZHANG $^{3,4}$, YIMEI SHU $^{5}$, QIUFENG QI $^{1,3}$, \\ MING ZHU ${ }^{1,3}$, YUN PENG ${ }^{1,3}$ and YANG LING $^{1,2}$ \\ ${ }^{1}$ Clinical Oncology Laboratory; ${ }^{2}$ Department of Oncology; ${ }^{3}$ Central Laboratory, Changzhou Tumor Hospital Affiliated to \\ Soochow University, Changzhou, Jiangsu 213032; ${ }^{4}$ Department of Clinical Medicine, The Third Affiliated Hospital of \\ Soochow University, Changzhou, Jiangsu 213003; ${ }^{5}$ Department of Clinical Medicine, \\ Nanjing Medical University, Nanjing, Jiangsu 211166, P.R. China
}

Received November 11, 2019; Accepted June 22, 2020

DOI: $10.3892 / \mathrm{ol} .2020 .11888$

\begin{abstract}
Immune checkpoint inhibitors (ICIs) have emerged as one of the most promising therapeutic options for patients with advanced cancer. The aim of the present study was to investigate the prognostic value of somatic mutations in mismatch repair (MMR) genes in metastatic cancers after ICI treatment, as well as their association with tumor mutational burden (TMB). Information regarding gene mutations in mismatch repair and the survival time of patients with advanced cancer following ICI treatment was collected from the cBioPortal database. The prognostic value of somatic mutations in MMR genes and the association between the mutation status and TMB score were analyzed among multiple types of cancer. Somatic mutation frequency in the MMR genes was identified to be $7 \%$ among all patients, which varied across different types of cancer. Somatic mutations in the MMR genes were associated with improved overall survival time in all tested patients $(\mathrm{P}=0.004)$. Following stratification by type of ICI treatment, a significant association was observed between somatic mutations in the MMR genes and overall survival time in patients treated with cytotoxic T-lymphocyte-associated protein 4 inhibitors $(\mathrm{P}=0.01)$. In addition, marked but non-significant association between somatic mutations in the MMR genes and overall survival time was revealed in patients administered with programmed death-1/programmed death-ligand-1 inhibitors $(\mathrm{P}=0.09)$. Multivariate Cox proportional hazards regression analysis demonstrated that somatic mutations
\end{abstract}

Correspondence to: Dr Yongping Liu, Clinical Oncology Laboratory, Changzhou Tumor Hospital Affiliated to Soochow University, 68 Honghe Road, Xinbei, Changzhou, Jiangsu 213032, P.R. China

E-mail: liuyongping026@126.com

Key words: somatic mutation, mismatch repair, immune checkpoint inhibitor, prognosis in MMR genes were significantly associated with overall survival time (hazard ratio, $0.683 ; 95 \%$ confidence interval, $0.497-0.938 ; \mathrm{P}=0.01)$. Patients with somatic mutations in the MMR genes demonstrated higher TMB compared with those not harboring mutations $(\mathrm{P}<0.01)$. The results of the present study suggested that somatic mutations in the MMR genes may be used as a prognostic marker of a positive outcome in patients with metastatic cancer receiving ICI treatment, since somatic mutations in the MMR genes may be one of the main factors affecting the tumor mutation load.

\section{Introduction}

Mismatch repair (MMR) deficiency (dMMR) has been used as a predictive biomarker to guide the clinical application of immune checkpoint inhibitor (ICI) therapy (1-3). dMMR mainly results from germline mutations and epigenetic alterations in any of the MMR genes, including MutL homologue 1 (MLH1), MutS protein homologue 2 (MSH2), MutS homologue 6 (MSH6) and PMS1 homologue 2 (PMS2) (4). In addition, dMMR can be caused by deletions in the epithelial cell adhesion molecule (EPCAM) gene, which leads to the inhibition of $M S H 2$ gene expression via promoter methylation (5). A previous study has reported that patients with cancer combined with dMMR and/or high microsatellite instability (MSI-H), who had no apparent germline mutations or MLH1 promoter hypermethylation, acquired somatic mutations in MMR genes, leading to a hypermutated tumor phenotype (6). Accumulating evidence supports the use of tumor mutational burden (TMB) as a biomarker for predicting the therapeutic response in patients with advanced cancer receiving ICIs, including antibodies that target cytotoxic $\mathrm{T}$ lymphocyte antigen 4 (CTLA-4) or programmed death 1/programmed death ligand 1 (PD-1/PD-L1) (7-10). TMB is associated with the production of new antigens, which may trigger antitumor immunity (11-13). A number of panels created based on targeted sequencing technology, such as the Memorial Sloan Kettering-Integrated Mutation Profiling of Actionable Cancer Targets (MSK-IMPACT) panel, have been approved by the US 
Food and Drug Administration (FDA) for the clinical evaluation of TMB $(14,15)$.

Hypermutation ability is an important feature of tumor development that may lead to the production of new antigens, which may be the result of functional defects in DNA repair, including that of dMMR $(16,17)$. Thus, it may be hypothesized that somatic mutations in genes associated with MMR may in turn affect MMR function, which may be of the underlying causes of the therapeutic effects exhibited by ICIs, making it a potential prognostic biomarker of ICI treatment. The Memorial Sloan Kettering Cancer Center (MSKCC) have disclosed their mutation data obtained from 1,661 patients with advanced cancer who received ICI treatment obtained by next generation sequencing (8), which are publicly available on the cBioPortal database. In the present study, these data were obtained with the aim of analyzing the prognostic value of somatic mutationsin genes involved in MMR in 1,661 patients with advanced cancer treated with ICIs; the potential association between somatic mutations in the MMR genes and TMB were also investigated.

\section{Materials and methods}

Data retrieval. Information regarding somatic mutations in genes associated with MMR and survival time in patients with advanced cancer who were treated with ICIs was downloaded from The Cancer Genome Atlas Database, an open access database that is publicly available at http://www.cbioportal. org $(18,19)$. The MSK-IMPACT Clinical Sequencing Cohort (https://www.cbioportal.org/study/summary?id=tmb_ mskcc_2018) was selected as the data source, containing only patients with advanced cancer who were administered with ICIs (8), and the number of samples was 1,661. Only data including mutations was obtained. The queried targeted genes included 4 main genes associated with MMR: $M L H 1$, MSH2, MSH6 and PMS2. Genomic and survival data from 1,661 patients with various types of cancer sequenced using the MSK-IMPACT assay were subsequently downloaded from the cBioPortal website after submitting the query' $M L H 1, M S H 2, M S H 6$ and $P M S 2^{\prime}$ in the input box. In total, 1,661 pieces of mutation and survival data, each containing the mutation status of the MMR genes, survival time and the status of patient (deceased or censored), were downloaded and analyzed. The TMB score was defined as the total number of nonsynonymous mutations in the somatic coding region which was normalized to the total number of megabases sequenced.

Statistical analysis. Statistical analyses were performed using the SPSS (version 17.0; SPSS, Inc.) and GraphPad Prism 7 (GraphPad Software, Inc.) software. The survival curves were produced using the Kaplan-Meier method and compared using log-rank tests. Overall survival (OS) data was obtained from the cBioPortal website. The definition of OS was the time between the date of first ICI treatment and the time of death or last follow-up visit (8). Hazard ratios of somatic mutations in the MMR genes for overall survival following adjustments for other prognostic factors was assessed using Cox proportional hazards regression. Unpaired t-test or one-way analysis of variance (ANOVA) was used for comparing the TMB scores between different groups, and the TMB scores were expressed as the mean $\pm \mathrm{SD}$. All P-values were two-sided. $\mathrm{P}<0.05$ was considered to indicate a statistically significant difference.

\section{Results}

Somatic mutation analysis of genes associated with MMR. The genomic profiles of four MMR genes, specifically $M L H 1$, MSH2, MSH6 and PMS2, in 1,661 patients were queried. Patients with any form of somatic mutations in these genes were assigned to the MMR mutation group. The queried genes were identified to be altered in 114 of the 1,661 patients; the total somatic mutation frequency of the MMR genes among all patients was $7 \%$. Variations were observed in the somatic mutation frequencies of the MMR genes among different types of tumors (Table I). Only patients with colorectal cancer possessed $>20 \%$ somatic mutations. Tumors with somatic mutation frequencies of $>7 \%$ included melanoma and cancer of unknown origin. Of note, patients with melanoma and somatic mutations in MMR exhibited superior survival benefits following ICI treatment in the present study.

Prognostic value of somatic mutations in the MMR genes in patients with metastatic cancer following ICI treatment. The prognostic value of somatic mutations in genes associated with MMR was first determined. Kaplan-Meier survival curves indicated that MMR gene mutation was a positive prognostic factor for OS. The median OS times for patients with MMR gene mutations and those in the wild-type group were 59.0 and 17.0 months, respectively ( $\mathrm{P}=0.004$; Fig. $1 \mathrm{~A})$, and the hazard ratio (HR) was 0.637 (95\% CI, 0.491-0.827), which was less than 1.0. Since mutations in the MMR genes include deleterious variants, variants of unknown significance and benign variants, the survival time in 114 cases were analyzed further; the Kaplan-Meier survival curve demonstrated that there was no significant difference in the OS time among the three types of mutations ( $\mathrm{P}=0.727$; data not shown).

The prognostic value of somatic mutations in the MMR genes in different types of tumors was next analyzed. Mutations in the MMR genes were significantly associated with prolonged survival in patients with melanoma $(\mathrm{P}=0.010$; Fig. 1B). In patients with colorectal and bladder cancer, the associations were not statistically significant, although the HRs were 0.608 (95\% CI, 0.294-1.256) and 0.578 (95\% CI, $0.271-1.235)$, respectively (data not shown).

To confirm the prognostic value of somatic mutations in genes associated with MMR in patients treated with ICIs, Cox proportional hazards regression analysis was performed. The presence of somatic mutations in the MMR genes was demonstrated to be significantly associated with OS following adjustment for age, sex, ICI treatment and tumor type (Table II).

Association between somatic mutations in the MMR genes and TMB score. To verify whether the presence of somatic mutations in MMR genes may affect tumor mutation load, analysis of TMB scores in groups of patients with different mutation status of the MMR genes was performed. Among all 1,661 patients, the TMB score in the MMR gene mutation group was significantly higher compared with that in the wild-type MMR gene group ( $\mathrm{P}<0.0001$; Fig. $2 \mathrm{~A})$. In the 114 patients with MMR gene mutations, the difference in 
Table I. Total somatic mutation frequency of MMR genes across different types of tumors.

\begin{tabular}{lccccccccccc}
\hline Type & CC & CUP & M & BLC & NSCLC & HNC & Glioma & EC & BC & RCC & SCNM \\
\hline Mutation frequency, \% & 21.82 & 7.95 & 7.5 & 6.98 & 4.86 & 6.47 & 5.13 & 4.76 & 4.54 & 1.99 & 100
\end{tabular}

MMR, mismatch repair; NSCLC, non-small cell lung cancer; M, melanoma; BLC, bladder cancer; RCC, renal cell carcinoma; HNC, head and neck cancer; EC, esophagogastric cancer; CC, colorectal cancer; CUP, cancer of unknown primary origin; BC, breast cancer; SCNM, skin cancer non-melanoma.

Table II. Hazard ratios for overall survival analysis.

\begin{tabular}{llcc}
\hline Variable & HR & 95\% CI & P-value \\
\hline $\begin{array}{l}\text { Mutation status of MMR } \\
\text { genes }\end{array}$ & & & \\
$\quad$ Wild-type (reference) & 1 & & \\
Mutation & 0.683 & $0.497-0.938$ & $0.018^{\mathrm{a}}$ \\
Treatment & & & $0.001^{\mathrm{a}}$ \\
CTLA-4 (reference) & 1 & & \\
PD-1/PDL-1 & 1.423 & $0.999-2.025$ & 0.050 \\
Combination & 1.528 & $1.219-1.914$ & $<0.001^{\mathrm{a}}$ \\
Age & & & $0.035^{\mathrm{a}}$ \\
$<30$ & 1 & & \\
31-50 & 0.871 & $0.582-1.304$ & 0.502 \\
51-60 & 0.648 & $0.435-0.966$ & $0.033^{\mathrm{a}}$ \\
61-70 & 0.708 & $0.476-1.052$ & 0.087 \\
$>71$ & 0.725 & $0.484-1.087$ & 0.120 \\
Tumor type & & & $<0.001^{\mathrm{a}}$ \\
Other (reference) & 1 & & \\
Bladder cancer & 0.901 & $0.628-1.294$ & 0.574 \\
Colorectal cancer & 0.575 & $0.427-0.774$ & $<0.001^{\mathrm{a}}$ \\
Melanoma & 1.305 & $1.025-1.661$ & $0.031^{\mathrm{a}}$ \\
Non-small lung cancer & 1.078 & $0.854-1.361$ & 0.529 \\
\hline
\end{tabular}

${ }^{\mathrm{a}} \mathrm{P}<0.05$. MMR, mismatch repair; HR, hazard ratio; $\mathrm{CI}$, confidence interval; CTLA-4, cytotoxic T lymphocyte antigen 4; PD-1/PD-L1, programmed death 1/programmed death ligand 1 .

the TMB scores among the three types of variants were not significant $(\mathrm{F}=0.6028 ; \mathrm{P}=0.5490)$ (one-way ANOVA; data not shown). Following stratification by type of cancer, the association between TMB score and MMR mutation status remained significant, and the TMB scores were higher in patients with MMR mutations, including melanoma $(\mathrm{t}=3.542 ; \mathrm{P}=0.0017)$, non-small cell lung cancer $(\mathrm{t}=2.550, \mathrm{P}=0.0230)$, colorectal cancer $(\mathrm{t}=6.533 ; \mathrm{P}<0.0001)$, bladder cancer $(\mathrm{t}=2.155, \mathrm{P}=0.0490)$ and other types of cancer $(\mathrm{t}=8.080 ; \mathrm{P}<0.0001)$ (Fig. 2B-F). The other types of cancer included renal cell carcinoma, head and neck cancer, esophagogastric cancer, glioma and cancer of unknown primary origin; somatic mutations in the MMR genes were infrequent in these types of tumors, the data from these patients were combined for this analysis.

The association between somatic mutations in MMR genes and TMB score in patients treated with different types of ICI
Table III. Top 10 genes with the highest frequency between the MMR gene mutation and wild-type groups.

\begin{tabular}{lccc}
\hline & \multicolumn{2}{c}{ Mutation frequency, $\%$} & \\
\cline { 2 - 3 } Gene & MMR mutation & MMR wild-type & P-value \\
\hline KMT2D & 50.00 & 11.57 & $1.50 \times 10^{-21}$ \\
NOTCH1 & 31.58 & 4.85 & $1.88 \times 10^{-17}$ \\
CREBBP & 28.95 & 4.72 & $2.39 \times 10^{-15}$ \\
RNF43 & 21.05 & 2.33 & $5.23 \times 10^{-14}$ \\
NOTCH3 & 25.44 & 4.14 & $1.68 \times 10^{-13}$ \\
KMT2C & 33.33 & 8.08 & $4.16 \times 10^{-13}$ \\
ARID1B & 23.68 & 3.62 & $4.27 \times 10^{-13}$ \\
ARID1A & 35.96 & 9.63 & $5.92 \times 10^{-13}$ \\
PTPRS & 22.81 & 3.36 & $6.55 \times 10^{-13}$ \\
GLI1 & 23.40 & 2.83 & $1.64 \times 10^{-12}$ \\
\hline
\end{tabular}

MMR, mismatch repair.

was subsequently analyzed. The TMB scores were significantly higher in patients with MMR mutations treated with the CTLA-4 inhibitor $(\mathrm{P}=0.0039)$, $\mathrm{PD}-1 / \mathrm{PD}-\mathrm{L} 1$ inhibitor $(\mathrm{P}<0.0001)$ and a combination of the two drugs $(\mathrm{P}=0.0096)$ compared with those in the corresponding wild-type groups (Fig. 3).

Co-mutation analysis of the MMR genes and other genes with the highest frequency. To clarify the reasons for the presence of somatic mutations in the MMR genesis associating with TMB, the profiles of genes with the high frequency of mutations alongside those of MMR were screened. A total of 286 genes were identified to be significantly co-mutated with somatic mutations in the MMR genes. Compared with those in the wild-type group, the top 10 gene mutation frequencies in the MMR gene mutation group were significantly increased (Table III).

\section{Discussion}

The application of ICIs has been beneficial to the survival of patients with tumors of certain molecular phenotypes, including tumors with MMR or a high TMB. Compared with patients with advanced cancer with a low TMB, a retrospective large-sample clinical study from the MSKCC has confirmed that patients with a high TMB were more likely to benefit from ICI treatment (8). In the present study, to clarify whether somatic mutations in the MMR genes may 
A

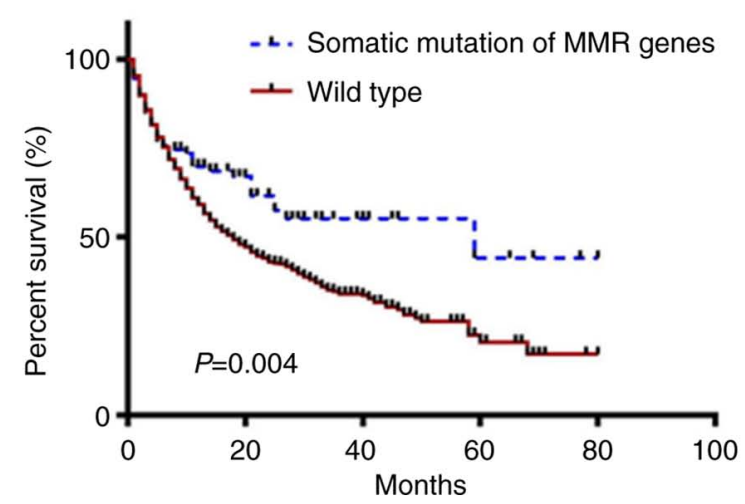

B

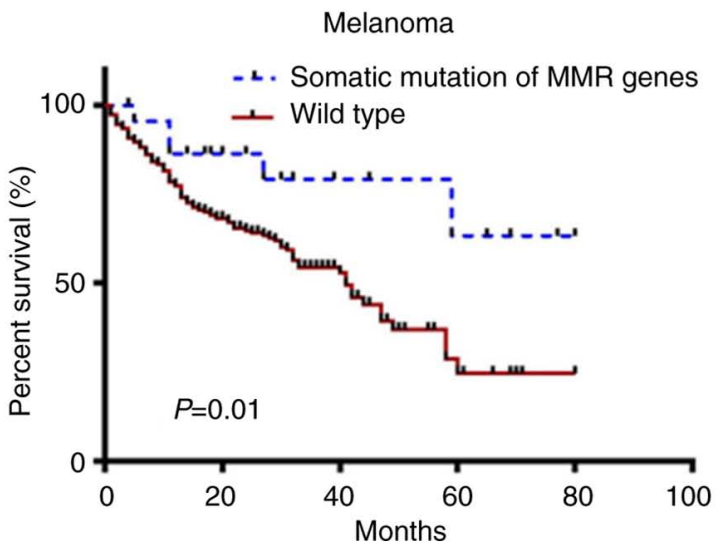

Figure 1. Association between the occurrence of somatic mutations in genes associated with MMR and patient survival. (A and B) Kaplan-Meier survival curves demonstrated that the presence of somatic mutations in MMR genes was associated with longer survival (A) in the entire cohort and (B) in patients with melanoma. MMR, mismatch repair.
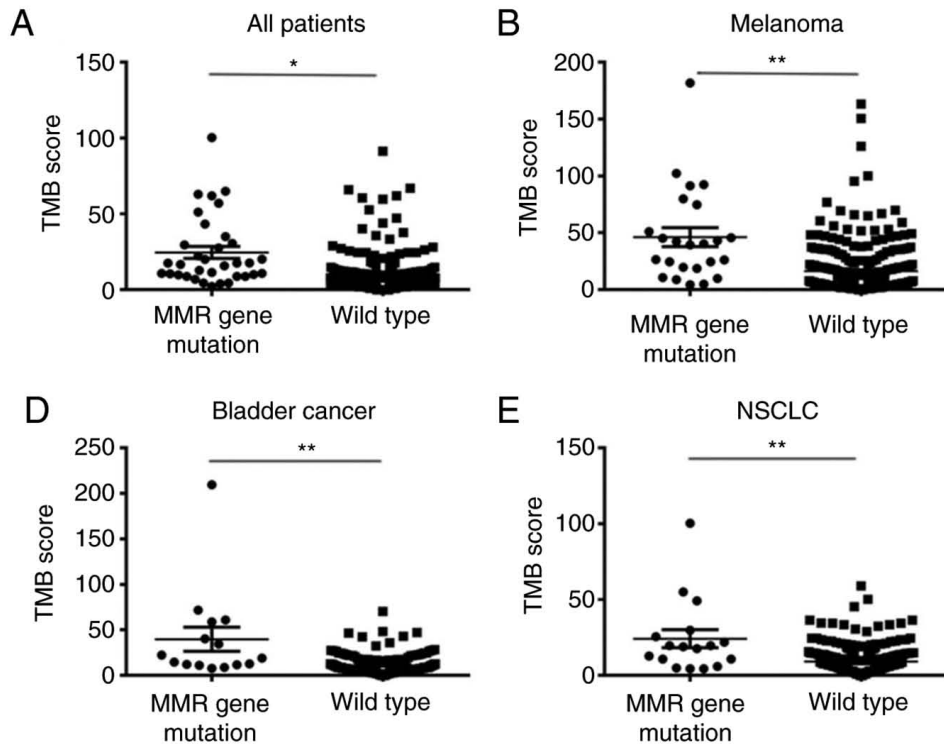

E

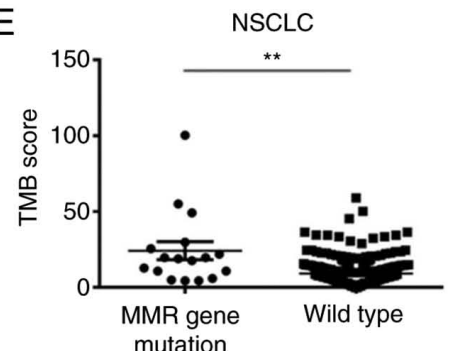

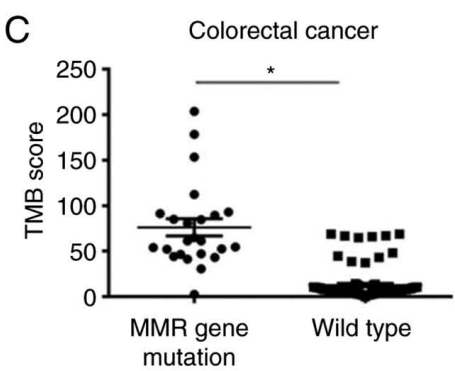

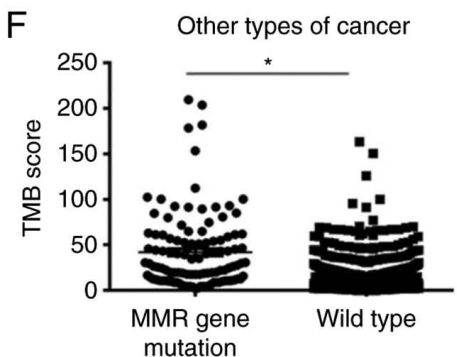

Figure 2. Association between the occurrence of somatic mutations in genes associated with MMR and TMB scores in patients with different types of cancer. (A-F) The TMB score in patients with somatic mutations in the MMR genes was significantly higher compared with that in the wild-type group (A) in all patients and (B) in melanoma (C) in colorectal cancer (D) in bladder cancer (E) in NSCLC and (F) in other types of cancer across most types of cancer. ${ }^{*} \mathrm{P}<0.0001,{ }^{* *} \mathrm{P}<0.01$. MMR, mismatch repair; TMB, tumor mutational burden.

be applied as a prognostic marker for patients receiving ICI treatment, relevant clinical and genomic data from the same cohort of patients treated with ICI were downloaded from the cBioPortal database. The association between the presence of somatic mutations in MMR genes and OS was analyzed, and the results demonstrated that somatic MMR gene mutations were significantly associated with improved OS in all patients with cancer, especially those with melanoma, compared with those with wild-type MMR genes. In addition, the TMB score was also found to be significantly higher in patients with MMR gene mutations compared with that in patients without MMR gene mutations. Somatic mutations in the MMR genes include three types of mutation variants, which are deleterious variants, variants of unknown significance and benign variants. It was demonstrated that there was no significant difference in the total survival times and the TMB scores among the three types of mutation variants.
The MMR system is mainly composed of four specific DNA MMR enzymes encoded by $M L H 1, P M S 2, M S H 2$ and $M S H 6$, the main function of which is to identify and repair mismatched bases during DNA replication. dMMR leads to microsatellite instability, susceptibility to cancer, accumulation of mutation burden of cancer-associated genes and the production of neoantigens, thus increasing the antitumor immune response. dMMR has been used as a potential biomarker inpredicting the responses of patients with solid tumors to ICIs, irrespective of cancer type $(2,20)$. dMMR mainly result from germline mutations, transcriptional inactivation of the aforementioned MMR genes through epigenetic alterations $(21,22)$, deletions in the EPCAM gene, leading to the constitutive repression of $\mathrm{MSH} 2$ expression by promoter methylation (5), or inflammation-mediated transcriptional repression (23). FDA has approved the use of a PD-1 antibody for the treatment of adult and pediatric patients with unresectable or metastatic 

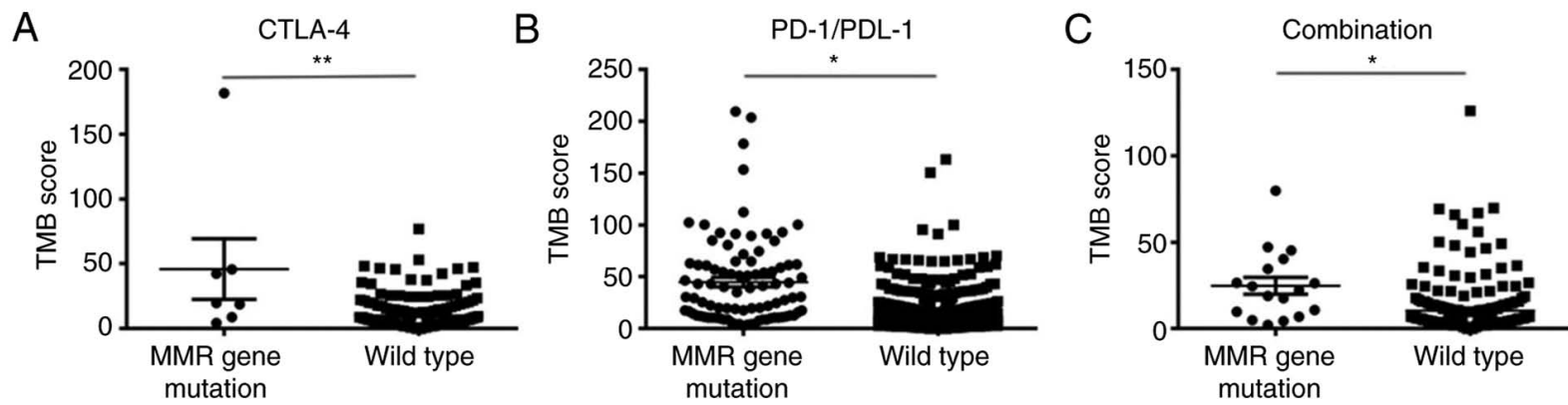

Figure 3. Association between the occurrence of somatic mutations in genes associated with MMR and TMB scores in patients treated with different types of immune checkpoint inhibitors. (A-C) The TMB scores were higher in patients with MMR gene mutations in patients treated with (A) the CTLA-4 inhibitor, (B) the PD-1/PDL-1 inhibitor and (C) a two-drug combination. ${ }^{*} \mathrm{P}<0.0001,{ }^{* *} \mathrm{P}<0.01$. MMR, mismatch repair; TMB, tumor mutational burden; CTLA-4, cytotoxic T lymphocyte antigen 4; PD-1, programmed death 1; PD-L1, programmed death-ligand 1.

solid tumors with MSI-H or dMMR (1). Since mutations in the $M L H 1, M S H 2, M S H 6$ and PMS2 genes appear to be the main causes of functional defects in MMR proteins (20), these four genes were selected for analysis in the present study. As showed in Table I, patients with colorectal cancer possessed $>20 \%$ somatic mutations. Tumors with somatic mutation frequencies of $>7 \%$ included melanoma and cancer of unknown origin. Of note, patients with somatic mutations of MMR in melanoma exhibited superior survival benefits following ICI treatment in the present study.

A previous study reported that although a number of patients with tumors with dMMR did not harbor germline mutations in the MMR genes, $70 \%$ of them had somatic mutations in genes associated with the MMR genes (6), suggesting this to be a potential cause of MSI-H or high TMB. Therefore, it may be hypothesized that somatic mutations in the MMR genes may serve as a novel prognostic biomarker for the outcome of ICI treatment. The present study demonstrated that the occurrence of somatic mutations in the MMR genes had a similar effect as TMB predicting the outcome of ICI treatment; it was not only associated with longer OS times in all patients treated with ICIs, but also in those with melanoma. Cox proportional hazards regression analysis revealed that somatic mutations in the MMR genes may serve as a prognostic indictor for longer OS in patients with cancer following ICI treatment. In addition to dMMR, MSI-H and high TMB scores being good predictors of ICI efficacy, the association between somatic mutations in genes associated with MMR and ICI efficacy may provide a new method for exploring the mechanism underlying the effects of dMMR on the efficacy of ICIs.

Somatic mutations in the MMR genes may be partly caused by dMMR due to the mismatched genes not being repaired in time, in turn increasing the TMB and tumor immunogenicity (6). The results of the present study demonstrated that somatic mutations in the MMR genes were associated with the TMB score. Following analysis of the entire cohort or in the stratified categories of patients with tumors treated with ICIs, the TMB score was revealed to be significantly higher in the group with MMR gene mutations compared with that in the wild-type group. This association remained significant in the different ICI treatment groups, suggesting that somatic mutations in the MMR genes may serve as a prognostic marker for the ICI treatments.
In addition to MMR, other types of DNA damage repair/response are also important for maintaining genomic integrity under normal cellular conditions (24). Failure to repair DNA damage can also result in a variety of genomic aberrations, leading to increased mutational load and neoantigen burden (25). However, this was beyond the scope of the present study and requires further analysis.

In the present study, a total of 286 genes were identified to be co-mutated with the MMR genes, which may be the result of dMMR. These co-mutations may be the underlying cause of the effects of MMR gene mutations on the TMB score. New antigens may be produced from the genetic mutations, which need to be further verified $(11,26)$. In the present study, the top 10 genes with the highest frequencies of mutation associated with MMR gene mutations have been listed in Table III. Histone-lysine N-methyltransferase 2D (KMT2D), belonging to a family of mammalian histone H3 lysine 4 (H3K4) methyltransferases, is frequently mutated in developmental diseases, such as in various forms of cancer (27). A study showed that ARID1A (the AT-rich interaction domain 1A, also known as BAF250a), KMT2D and SOX9 is sufficient to detect mismatch repair-deficient colorectal adenocarcinomas with $76 \%$ sensitivity and $98 \%$ specificity (28). ARID1A is one of the most commonly mutated genes in cancer, and its deficiency is correlated with microsatellite instability and increased mutation load across multiple human cancer types (29). Notch1 inhibition reduces immune-suppressive cells. The inhibition of Notch1 improves the antitumor activity of nivolumab and ipilimumab, particularly when given in combination (30). One gene implicated in ALL relapse encodes cyclic adenosine monophosphate (cAMP) response element binding protein (CREB) binding protein (CREBBP/CBP), a member of the KAT3 family of histone acetyltransferases (HAT). CREBBP knockdown enhances RAS/RAF/MEK/ERK signaling in Ras pathway mutated acute lymphoblastic leukemia but does not modulate chemotherapeutic response (31). Mutation of ARID1A and CREBBP may be poor prognostic factors for follicular lymphoma receiving immunochemotherapy (32). Genomic alteration frequency of ring finger protein 43 (RNF43) are higher in PD-L1-positive patients with biliary tract cancer (BTC) compared with PD-L1-negative patients, which suggest that mutation status of RNF43 may be a poor prognostic factor for BTC receiving immunotherapy (33). 
Treg cell expansion requires Notch3 signaling, which imply that the mutation status of Notch3 may affect the function of Treg cells (34). NOTCH1/2 and KMT2C/D were the most frequently mutated genes in recurrent or metastatic skin squamous cell cancer (35). PTPRS, a receptor-type protein tyrosine phosphatase, appears to regulate RAS pathway activation through ERK. Native mutations in PTPRS, may reduce its phosphatase activity while increasing ERK activation and downstream transcriptional signaling (36). Glioma oncogene homolog 1 (GLI1) is an oncogenic transcription factor playing important roles in cancer. Patients with GLI1 activation and breast tumors had worse metastasis-free survival compared with those with low levels (37). The association between the aforementioned gene variants and the efficacy of immunologic checkpoint inhibitors is not clear and needs to be further verified. However, the role of these mutated genes in MMR and the possible underlying mechanisms in regulating the efficacy of ICIs remain to be fully elucidated.

In the present study, the effects of chemotherapy and radiotherapy on the dMMR involvement in predicting the efficacy of ICIs were not examined. The majority of patients with advanced cancer receive multiple courses of radiotherapy and chemotherapy, which may affect the increase in TMB and the efficacy of ICIs; this requires further exploration.

In a conclusion, the results of the present study suggested that somatic mutations in the MMR genes may be used as a prognostic marker of a positive outcome in patients with metastatic cancer receiving ICI treatment; since somatic mutations in the MMR genes may be one of the main factors affecting the tumor mutation load.

\section{Acknowledgements}

Not applicable.

\section{Funding}

The present study was supported by the Science and Technology Planning Project of Changzhou, Jiangsu Province (grant no. CE20165052), the Changzhou Health Bureau Project (grant no. ZD201616), the Jiangsu Province Health Department Project (grant no. Z201616), the 333 Talents Training Project of Jiangsu Province (grant nos. 2016 III-0727 and BRA2017114) and the Talents Training Project for Key Medical Innovation of Changzhou (grant no. 2016CZLJ021).

\section{Availability of data and materials}

The datasets generated and/or analyzed during the present study are available in The Cancer Genome Atlas database, an open access database that is publicly available at http://www.cbioportal. org.

\section{Authors' contributions}

YPL conceived the study and wrote the manuscript. YL participated in the study design. LC and SLZ analyzed and interpreted a part of the data. YMS interpreted part of the data. QFQ, MZ and YP analyzed a part of the data. All authors read and approved the final manuscript.

\section{Ethics approval and consent to participate}

Not applicable.

\section{Patient consent for publication}

Not applicable.

\section{Competing interests}

The authors declare that they have no competing interests.

\section{References}

1. Lemery S, Keegan P and Pazdur R: First FDA approval agnostic of cancer site-when a biomarker defines the indication. N Engl J Med 377: 1409-1412, 2017.

2. Le DT, Uram JN, Wang H, Bartlett BR, Kemberling $H$, Eyring AD, Skora AD, Luber BS, Azad NS, Laheru D, et al: PD-1 Blockade in tumors with mismatch-repair deficiency. N Engl J Med 372: 2509-2520, 2015.

3. Le DT, Durham JN, Smith KN, Wang H, Bartlett BR, Aulakh LK, Lu S, Kemberling H, Wilt C, Luber BS, et al: Mismatch repair deficiency predicts response of solid tumors to PD-1 blockade. Science 357: 409-413, 2017.

4. Mills AM, Liou S, Ford JM, Berek JS, Pai RK and Longacre TA: Lynch syndrome screening should be considered for all patients with newly diagnosed endometrial cancer. Am J Surg Pathol 38: 1501-1509, 2014

5. Giardiello FM, Allen JI, Axilbund JE, Boland CR, Burke CA, Burt RW, Church JM, Dominitz JA, Johnson DA, Kaltenbach T, et al: Guidelines on genetic evaluation and management of Lynch syndrome: A consensus statement by the US Multi-society task force on colorectal cancer. Am J Gastroenterol 109: 1159-1179, 2014.

6. Haraldsdottir S, Hampel H, Tomsic J, Frankel WL, Pearlman R, de la Chapelle A and Pritchard CC: Colon and endometrial cancers with mismatch repair deficiency can arise from somatic, rather than germline, mutations. Gastroenterology 147: 1308-1316.e1, 2014.

7. Wang F, Wei XL, Wang FH, Xu N, Shen L, Dai GH, Yuan XL, Chen Y, Yang SJ, Shi JH, et al: Safety, efficacy and tumor mutational burden as a biomarker of overall survival benefit in chemo-refractory gastric cancer treated with toripalimab, a PD-1 antibody in phase Ib/II clinical trial NCT02915432. Ann Oncol 30: 1479-1486, 2019.

8. Samstein RM, Lee CH, Shoushtari AN, Hellmann MD, Shen R, Janjigian YY, Barron DA, Zehir A, Jordan EJ, Omuro A, et al: Tumor mutational load predicts survival after immunotherapy across multiple cancer types. Nat Genet 51: 202-206, 2019.

9. Hellmann MD, Ciuleanu TE, Pluzanski A, Lee JS, Otterson GA, Audigier-Valette C, Minenza E, Linardou H, Burgers S, Salman P, et al: Nivolumab plus ipilimumab in lung cancer with a high tumor mutational burden. N Engl J Med 378: 2093-2104, 2018.

10. Yi M, Jiao D, Xu H, Liu Q, Zhao W, Han X and Wu K: Biomarkers for predicting efficacy of PD-1/PD-L1 inhibitors. Mol Cancer 17: 129,2018

11. Gubin MM, Artyomov MN, Mardis ER and Schreiber RD: Tumor neoantigens: Building a framework for personalized cancer immunotherapy. J Clin Invest 125: 3413-3421, 2015.

12. Schumacher TN, Kesmir C and van Buuren MM: Biomarkers in cancer immunotherapy. Cancer Cell 27: 12-14, 2015.

13. Grizzi G, Caccese M, Gkountakos A, Carbognin L, Tortora G, Bria $E$ and Pilotto S: Putative predictors of efficacy for immune checkpoint inhibitors in non-small-cell lung cancer: Facing the complexity of the immune system. Expert Rev Mol Diagn 17: 1055-1069, 2017.

14. Zehir A, Benayed R, Shah RH, Syed A, Middha S, Kim HR, Srinivasan P, Gao J, Chakravarty D, Devlin SM, et al: Mutational landscape of metastatic cancer revealed from prospective clinical sequencing of 10,000 patients. Nat Med 23: 703-713, 2017.

15. Chalmers ZR, Connelly CF, Fabrizio D, Gay L, Ali SM, Ennis R, Schrock A, Campbell B, Shlien A, Chmielecki J, et al: Analysis of 100,000 human cancer genomes reveals the landscape of tumor mutational burden. Genome Med 9: 34, 2017. 
16. Hanahan D and Weinberg RA: Hallmarks of cancer: The next generation. Cell 144: 646-674, 2011.

17. Burrell RA, McGranahan N, Bartek J and Swanton C: The causes and consequences of genetic heterogeneity in cancer evolution. Nature 501: 338-345, 2013.

18. Cerami E, Gao J, Dogrusoz U, Gross BE, Sumer SO, Aksoy BA, Jacobsen A, Byrne CJ, Heuer ML, Larsson E, et al: The cBio cancer genomics portal: an open platform for exploring multidimensional cancer genomics data. Cancer Discov 2: 401-404, 2012.

19. Gao J, Aksoy BA, Dogrusoz U, Dresdner G, Gross B, Sumer SO Sun Y, Jacobsen A, Sinha R, Larsson E, et al: Integrative analysis of complex cancer genomics and clinical profiles using the cBioPortal. Sci Signal 6: pl1, 2013.

20. Marcus L, Lemery SJ, Keegan P and Pazdur R: FDA approval summary: Pembrolizumab for the treatment of microsatellite instability-high solid tumors. Clin Cancer Res 25: 3753-3758, 2019.

21. Seth S, Ager A, Arends MJ and Frayling IM: Lynch syndrome-cancer pathways, heterogeneity and immune escape. J Pathol 246: 129-133, 2018.

22. Lynch HT, Snyder CL, Shaw TG, Heinen CD and Hitchins MP: Milestones of Lynch syndrome: 1895-2015. Nat Rev Cancer 15 181-194, 2015.

23. Eso Y, Takai A, Matsumoto T, Inuzuka T, Horie T, Ono $\mathrm{K}$, Uemoto S, Lee K, Edelmann W, Chiba T and Marusawa $\mathrm{H}$ $\mathrm{MSH} 2$ dysregulation is triggered by proinflammatory cytokine stimulation and is associated with liver cancer development. Cancer Res 76: 4383-4393, 2016.

24. Arora S, Velichinskii R, Lesh RW, Ali U, Kubiak M, Bansal P, Borghaei H, Edelman MJ and Boumber Y: Existing and emerging biomarkers for immune checkpoint immunotherapy in solid tumors. Adv Ther 36: 2638-2678, 2019.

25. Teo MY, Seier K, Ostrovnaya I, Regazzi AM, Kania BE, Moran MM, Cipolla CK, Bluth MJ, Chaim J, Al-Ahmadie H, et al: Alterations in DNA damage response and repair genes as potential marker of clinical benefit from PD-1/PD-L1 blockade in advanced urothelial cancers. J Clin Oncol 36: 1685-1694, 2018.

26. Türeci Ö, Löwer M, Schrörs B, Lang M, Tadmor A and Sahin U: Challenges towards the realization of individualized cancer vaccines. Nat Biomed Eng 2: 566-569, 2018.

27. Froimchuk E, Jang Y and Ge K: Histone H3 lysine 4 methyltransferase KMT2D. Gene 627: 337-342, 2017.

28. Lv J, Zhu Y, Ji A, Zhang Q and Liao G: Mining TCGA database for tumor mutation burden and their clinical significance in bladder cancer. Biosci Rep 40: BSR20194337, 2020.

29. Shen J, Ju Z, Zhao W, Wang L, Peng Y, Ge Z, Nagel ZD, Zou J, Wang C, Kapoor P, et al: ARID1A deficiency promotes mutability and potentiates therapeutic antitumor immunity unleashed by immune checkpoint blockade. Nat Med 24: 556-562, 2018.
30. Qiu H, Zmina PM, Huang AY, Askew D and Bedogni B: Inhibiting Notch1 enhances immunotherapy efficacy in melanoma by preventing Notch1 dependent immune suppressive properties. Cancer Lett 434: 144-151, 2018.

31. Dixon ZA, Nicholson L, Zeppetzauer M, Matheson E, Sinclair P, Harrison CJ and Irving JA: CREBBP knockdown enhances $\mathrm{RAS} / \mathrm{RAF} / \mathrm{MEK} / \mathrm{ERK}$ signaling in Ras pathway mutated acute lymphoblastic leukemia but does not modulate chemotherapeutic response. Haematologica 102: 736-745, 2017.

32. Pastore A, Jurinovic V, Kridel R, Hoster E, Staiger AM, Szczepanowski M, Pott C, Kopp N, Murakami M, Horn H, et al: Integration of gene mutations in risk prognostication for patients receiving first-line immunochemotherapy for follicular lymphoma: A retrospective analysis of a prospective clinical trial and validation in a population-based registry. Lancet Oncol 16: 1111-1122, 2015.

33. Mody K, Starr J, Saul M, Poorman K, Weinberg BA, Salem ME, VanderWalde A and Shields AF: Patterns and genomic correlates of PD-L1 expression in patients with biliary tract cancers. J Gastrointest Oncol 10: 1099-1109, 2019.

34. Kared H, Adle-Biassette H, Foïs E, Masson A, Bach JF, Chatenoud L, Schneider E and Zavala F: Jagged2-expressing hematopoietic progenitors promote regulatory $\mathrm{T}$ cell expansion in the periphery through notch signaling. Immunity 25: 823-834, 2006.

35. Cavalieri S, Perrone F, Miceli R, Ascierto PA, Locati LD, Bergamini C, Granata R, Alfieri S, Resteghini C, Galbiati D, et al: Efficacy and safety of single-agent pan-human epidermal growth factor receptor (HER) inhibitor dacomitinib in locally advanced unresectable or metastatic skin squamous cell cancer. Eur J Cancer 97: 7-15, 2018.

36. Davis TB, Yang M, Schell MJ, Wang H, Ma L, Pledger WJ and Yeatman TJ: PTPRS regulates colorectal cancer RAS pathway activity by inactivating Erk and preventing its nuclear translocation. Sci Rep 8: 9296, 2018.

37. Sirkisoon SR, Carpenter RL, Rimkus T, Anderson A, Harrison A, Lange AM, Jin G, Watabe K and Lo HW: Interaction between STAT3 and GLI1/tGLI1 oncogenic transcription factors promotes the aggressiveness of triple-negative breast cancers and HER2-enriched breast cancer. Oncogene 37: 2502-2514, 2018.

This work is licensed under a Creative Commons Attribution-NonCommercial-NoDerivatives 4.0 International (CC BY-NC-ND 4.0) License. 